\title{
Wide triplets of galaxies
}

\author{
A. D. Chernin ${ }^{1,2}$, V. P. Dolgachev ${ }^{1}$ and L. M. Domozhilova ${ }^{1}$ \\ ${ }^{1}$ Sternberg Astronomical Institute, Moscow University, Moscow, 119899, \\ Russia \\ ${ }^{2}$ Tuorla Observatory, University of Turku, Piikkiö, 21 500, Finland
}

\begin{abstract}
Triple systems of galaxies with the typical mass $\sim 10 h^{13} M \odot$ and crossing time about the Hubble time provide evidence for ongoing collapse on the space scale $\sim 1 \mathrm{Mpc}$. This is confirmed by a set of computer simulations with the use of free-fall models.
\end{abstract}

\section{Introduction}

The origin and evolution of structure on different mass and space scales is one of the outstanding issues in cosmology. There is clear evidence that galaxy clusters of $\sim 10^{15}-10^{16} M \odot$ are evolving via collapse, clustering and accretion of smaller groups (see, for instance, White et al., 1993, and references therein). However it is less certain whether groups of $\sim 10^{12}-10^{14} M \odot$ also evolve in the same way.

In this paper, we study small groups of galaxies which seem to be in a state of ongoing collapse. These are wide triplets of galaxies with masses $\sim 10^{13} M \odot$ and space scale $\sim 1 \mathrm{Mpc}$.

\section{Basic data}

A list of wide triple systems of galaxies was compiled by Trofimov and Chernin (1995) with the use of the data from the catalogues by Geller and Huchra (1983) (the Northern sky) and Maia et al. (1989) (the Southern sky). The list contains 108 systems; 38 of them can be considered isolated physical systems, according to a general criterion suggested by Anosova (1988).

The major statistical (median) characteristics of 38 physical systems are as follows: the projected $\mathrm{rms}$ velocity dispersion $V=84 \mathrm{~km} \mathrm{~s}^{-1}$, projected mean harmonic separation $R=556 h^{-1} \mathrm{kpc}$, dimensionless crossing time $\tau=0.95$, virial mass $M_{v i r}=1.5 \times 10^{12} h^{-1} M \odot$, calculated with the use of a 'standard' estimator (Binney and Tremaine 1987). The Hubble constant is normalized here to $75 \mathrm{~km} \mathrm{~s}^{-1} \mathrm{Mpc}^{-1}$.

We call the systems wide triplets, in contrast to compact triplets of galaxies with $R=43 \mathrm{kpc}$, studied in detail by Karachentseva et al. (1979) and Karachentsev et al. (1989).

Compact and wide triplets differ from each other in a number of ways. Most obvious is the difference in size - more than an order of magnitude. The sizes of compact systems are so small that they are comparable to or likely less than 
the radii of extended dark matter halos around giant galaxies. On the contrary, in wide triplets, the separations between the member galaxies are considerably larger than the radii of the galaxy halos; this means in particular that a threebody treatment is adequate for these systems, at least in the first approximation - see Sec.3.

The dimensionless crossing time is $\tau \approx 0.1$ for the compact systems, while $\tau \approx 1$ for the wide ones. The galaxies in compact triplets have made several crossings during their life-time. This might be enough to reach virial equilibrium in the systems and their ensemble. In contrast to this, $\tau \sim 1$ for the wide triplet ensemble means that the systems are dynamically young; they are far from a virialized state. Most probably, wide triplets are in the state of ongoing collapse. Our computer simulations confirm this conclusion.

\section{Computer simulations}

An analysis of the dynamical and evolutionary state of wide triplets was first made by Dolgachev and Chernin (1997). Here we present recent results of computer simulations of the systems based on an assumption that the member galaxies of the systems were at rest some $12 \pm 2 \mathrm{Gyr}$ ago. This is essentially the same physical conjecture as that of the model by Kahn and Voltjer (1959) for the Local Group. In the Kahn-Voltjer model, there was a moment in the past, 10-20 Gyr ago, when the centers of masses of two major galaxies of the Local Group were at rest after an earlier stage when the masses moved apart due to the whole Hubble expansion; since that moment, the galaxies have started to move towards each other under the action of their mutual gravity.

The models with zero initial velocities are usually called free-fall models. We also assume that the free-fall of galaxies in the wide triplets can be treated as the motion of point masses (see above), and we take equal masses of the bodies in each system, for simplicity. A soften-potential code was used which is similar to one worked out by Aarseth (1971).

About 6000 simulated dynamical states were computed for various total masses $M$ of the systems and their initial size $L$. Among them, 1320 states with $M=9 \times 10^{12} M \odot$ and $R_{0}=1.4-3 \mathrm{Mpc}$ were found to reproduce the observational data most closely. They were used for statistical analysis, and, with random number generator, 10 samples were produced with 50 states in each selected from this variety of states. It proves that the difference between the observed figures of $V, R, \tau$ and the dynamical characteristics given by the computer statistics does not exceed 10-20\%. This seems to be considered a reasonable accuracy, if one takes into account usual observational errors, especially in velocity measurements for galaxies in groups (see the catalogues by Geller and Huchra (1983) and Maia et al. 1989).

Not only median values of $V, R$ and $\tau$, but also the distributions of these quantities in the computer statistics demonstrate a rather high degree of similarity with the observed distributions (more details see in Chernin et al. 2000). 


\section{Conclusions}

Our analysis of the observational data, as well as our computer simulations, demonstrate clearly that wide triplets of galaxies present a class of systems which are in the state of ongoing collapse. Many (if not all) bright galaxies of wide triplets have smaller satellite galaxies, so that their collapse is actually the collapse of subgroups. In this way, wide triplets provide new direct cosmological constraints by establishing that hierarchical evolution is occurring on a scale of $\sim 1 \mathrm{Mpc}$.

The typical mass of a wide triplet estimated statistically on the basis of the simulations is $M \sim 10^{13} M_{\odot}$. This is an order of magnitude larger than the virial mass estimated for these systems with the use of a 'standard' formula (Sec. 2). This is a quantitative evidence that wide triplets are very far from virial equilibrium.

The mass of a typical wide triplet is about 3 times larger than the mass of the Local Group as estimated by the Kahn-Voltjer model. This means that three-body dynamics can 'hide' a larger mass than two-body dynamics behind similar visible sizes and velocities.

The mass of a typical wide triplet proves to be close to the total mass obtained for a dozen small compact groups with the use of X-ray data (Mulchaey 1995). The compact groups are assumed to be in virial and thermal equilibrium, and they are similar to compact triplets. Because of this one can expect that the total mass of a compact triplet (including their extended halo) is also $\sim 10^{13} M \odot$. In both wide and compact triplets, the major contribution to the total mass is obviously due to dark matter. The corresponding mass-to-light ratio can be estimated as $(M / L) \approx(150-200) h \times\left(M_{\odot} / L_{\odot}\right)$. This figure indicates that dark matter mass is 15-30 times the mass of baryonic matter in the triplets of both types.

Wide triplets and compact triplets reveal that group evolution on a given mass scale may proceed in various ways: triplets of these types have different space scales and different physical states. With the use of our computer models, one can see that wide triplets can reach the state of virial equilibrium in not less than 20-30 Gyr. They cannot, therefore, be considered as compact triplets at their initial stage of evolution. Compact triplets evolved more rapidly, and this may be possible only, if the initial (at the moment of the start of collapse) dark matter density was higher in these systems than in wide systems. This means that the cosmological density contrast might be essentially different on a given mass scale, depending on the location of the systems. Most probably, wide systems form in the outer areas of clusters and superclusters, while the compact ones forms in their inner areas.

Though the free-fall models we have developed simulate the observed data on wide triple systems with reasonable accuracy, a question arises: Is this type of model the only one possible? A drastic challenge to the free-fall models is presented by a sophisticated theory developed recently for the Local Group as an alternative to the Kahn-Voltjer model. Valtonen et al. $(1993,1995)$ and Byrd et al. (1994) argued that the pre-history of the Local Group might be full of violent events, including close passages, collisions and even merging of galaxies. It is also important that all this complex evolution does not need any considerable mass excess in the volume of the group. Perhaps an adaptation of this approach to 
wide triplets would give rise to results that would look very different from what was discussed above. Were close passages, collisions and merging of galaxies possible in the early evolution of these systems? How frequently might they occur? Can any signs of these violent processes be found in observations? These questions remain open.

Acknowledgments. We thank A. Trofimov for cooperation and G. Byrd, J. Einasto, I. Karachentsev, and M. Valtonen for useful discussions. Partial support from Russian Foundation for Basic Research is appreciated.

\section{References}

Aarseth, S. J. 1963, MNRAS, 126, 223

Anosova, J. P. 1988, Astrophysics 26-27, 653

Binney, J. \& Tremaine, S. 1987 Galactic Dynamics, Princeton University Press Byrd, G., Valtonen, M., McCall, M. \& Innanen, K. 1994, AJ 107, 2055

Chernin, A. D., Dolgachev, V. P., Domozhilova, L. M. 2000 (in press)

Dolgachev, V. P. \& Chernin, A.D. 1997, Astron. Rep., 41, 284

Geller, M. J. \& Huchra J. P. 1983, ApJS, 52, 61

Kahn, F. D. \& Woltjer, L. 1959, ApJ, 130, 705

Karachentsev, I. D., Karachentseva, V. E., \& Lebedev, V. S. 1989, Izvestija $\mathrm{SAO}, 27,67$

Karachentseva, V. E., Karachentsev, I. D., \& Shcherbanivskii, A. L. 1979, Izvestija SAO, 11, 3

Maia, M. A. G., da Costa, L. N., \& Latham, D. W. 1989, ApJS, 69, 809

Mulchaey J. S., 1995, in in Dark Matter, AIP Conference Proceedings, 336, eds.

S. S. Holt and C. L. Bennett, American Institute of Physics - New York, p. 243

Mulchaey J. S., Zabludoff A. I., 1998, ApJ, 496, 73

Trofimov, A. V. \& Chernin, A. D. 1995, Astron. Rep., 39, 308

Valtonen, M. J., Byrd, G. G., McCall, M. L. \& Innanen, K. A. 1993, AJ 105, 886

Valtonen, M. J., McCall, M. L., Innanen, K. A., Zheng, J.-Q. \& Byrd, G. G. 1995, in Dark Matter, AIP Conference Proceedings, 336, eds. S. S. Holt and C. L. Bennett, American Institute of Physics - New York, p. 450

White S. D. M., Briel U., Henry J. P., 1993, MNRAS, 261, L8 